\title{
Transcriptional Profiles in Ischemia/Reperfusion- Injured Murine Kidneys Synergistically Protected by Erythropoietin Derived Peptide CHBP and Caspase-3 SiRNA
}

\section{Yuanyuan Wu}

Nantong University https://orcid.org/0000-0002-6253-7773

\section{Weiwei Chen}

Leicester-Nantong Joint Institute of Kidney Science, Department of Nephrology, Affiliated Hospital of Nantong University, China.

\section{Yufang Zhang}

Renal Group, Basic Medical Research Centre, Nantong University, China.

\section{Aifen Liu}

Renal Group, Basic Medical Research Centre, Nantong University, China.

\section{Cheng Yang}

Department of Urology, Zhongshan Hospital, Fudan University; Shanghai Key Laboratory of Organ

Transplantation, Shanghai, China.

\section{Hui Wang}

Leicester-Nantong Joint Institute of Kidney Science, Department of Nephrology, Affiliated Hospital of Nantong University, China.

\section{Tongyu Zhu}

Department of Urology, Zhongshan Hospital, Fudan University; Shanghai Key Laboratory of Organ Transplantation, Shanghai, China.

\section{Yaping Fan}

Leicester-Nantong Joint Institute of Kidney Science, Department of Nephrology, Affiliated Hospital of Nantong University, China.

\section{Bin Yang ( $\sim$ by5@le.ac.uk)}

Renal Group, Department of Cardiovascular Sciences, University of Leicester; University Hospitals of Leicester, United Kingdom. https://orcid.org/0000-0001-5315-9240

\section{Research}

Keywords: ischemia/reperfusion injury, CHBP, small interference RNA (siRNA), caspase-3 
DOI: https://doi.org/10.21203/rs.3.rs-26803/v1

License: (c) (1) This work is licensed under a Creative Commons Attribution 4.0 International License. Read Full License

Version of Record: A version of this preprint was published at Journal of Pharmacology and Experimental Therapeutics on August 5th, 2020. See the published version at https://doi.org/10.1124/jpet.120.000092. 


\section{Abstract}

Background: Target-specific treatment is not available for acute kidney injury (AKI). A novel erythropoietin-derived cyclic helix B surface peptide (CHBP) protects kidneys against AKI subjected to different causes. Herein, we investigated the transcriptional profile of renoprotection induced by $\mathrm{CHBP}$ and its potential synergistic effects with caspase-3 siRNA (CASP3siRNA) on ischemia/reperfusion (IR) injury associated AKI.

Methods: A mouse renal IR model was established by clamping bilateral pedicles for 30 min and reperfusion for 48 h. $0.03 \mathrm{mg} / \mathrm{kg}$ of CASP3siRNA/negative control (NCsiRNA) was injected via tail vein 2 $\mathrm{h}$ pre-surgery, with/without $24 \mathrm{nmol} / \mathrm{kg}$ of CHBP administered to peritoneal cavity at 15 min post reperfusion. The transcriptomic profile in kidneys was assessed by affymetrix gene chips, along with renal function, histology, active caspase-3 and HMGB1.

Results: CHBP or CASP3siRNA significantly improved renal function and structure, with decreased caspase-3 and HMGB1 in IR kidneys. Combined treatment of CHBP and CASP3siRNA further preserved kidney structure, and reduced active caspase-3 and HMGB1. Furthermore, fold change $>1.414$ and $P<$ 0.05 were used to identify differentially expressed genes (DEGs). In IR kidneys, 281 DEGs induced by CHBP were mainly involved in promoting cell division and improving cellular function and metabolism (up-regulated STAT5B and SLC22A7). The additional administration of CASP3siRNA caused 504 and 418 DEGs in IR + CHBP kidneys with or without NCsiRNA, with 37 genes in common. These DEGs were associated with modulated apoptosis and inflammation (up-regulated BCL6囚SLPI and SERPINA3M), and immunity, injury and microvascular homeostasis (up-regulated CFH and GREM1, and down-regulated ANGPTL2).

Conclusions: This proof-of-effect study indicated that the synergistic renoprotection of CHBP and CASP3siRNA at the early stage of IR-induced AKI. Underlying genes, BCL6, SLPI, SERPINA3M, GREM1 and ANGPTL2, might be potential new biomarkers for clinical applications.

\section{Introduction}

Acute kidney injury (AKI) is a public health problem and has attracted much attention in recent years [1]. In worldwide, AKI affects about $2 \%$ patients in hospital admissions with a rate of mortality about $12 \%$, both of which were increased to around $20 \%$ in the intensive care unit $[2,3]$. There is no specific treatment for AKI apart from passive support or renal replacement therapy such as volume control or dialysis in clinic [4]. It is urgent, therefore, to develop specific and effective treatment for AKI to reduce mortality and prevent its progression to chronic kidney disease $[5,6]$.

Renal ischemia/reperfusion (IR) injury is a major cause of AKI, characterized by apoptosis, inflammation and immune responses associated damage $[7,8]$. Recently, the innate repair mechanism in AKI has attracted great attention, which is highlighted by an innate repair receptor, a heterodimer of erythropoietin (EPO) receptor and $\beta$ common receptor (EPOR/ $\beta c R)$ [9]. EPO, a natural ligand of $E P O R / \beta c R$, is defective in 
tissue protection due to low affinity, but high affinity to a homodimer receptor (EPOR) ${ }_{2}$ in erythropoiesis [10-12]. EPO-derived helix B surface peptide (HBSP) and cyclic HBSP (CHBP, more stable and potent than HBSP [13]) only bind with $E P O R / \beta c R$, so remaining the tissue protective property without erythropoiesis, and have promising potential for clinical application [14-17]. In the IR kidney, CHBP reduces endoplasmic reticulum stress [18] and increases autophagy [13], leading to less apoptosis [19]. CHBP also ameliorated renal inflammation and reduced chronic deposition of extracellular matrix through inactivating forkhead box 03 a (FoxO3a) after IR [20]. Nevertheless, the exact underling mechanism in the renoprotection of CHBP is incompletely understood.

Caspase-3, up-regulated by IR in the kidney, is a major effector enzyme in the process of apoptosis, as well as inflammation [21, 22]. Evidence suggests that down-regulating the expression of active caspase-3 is presented by HBSP/CHBP treatment in IR kidneys $[17,23]$. The contributing role of caspase-3 in IR kidneys was further verified by small interfering RNA (siRNA), showing that serum-stabilized siRNA targeting caspase-3 greatly reversed renal function and inflammation in a 2-week porcine kidney autotransplantation model [24]. It is also intriguing to discover whether there are synergistic effects on IRinduced AKI by combined administration of HBSP/CHBP and caspase-3 siRNA (CASP3siRNA).

In the present study, the effect of CHBP was explored by a single peritoneal injection, as well as its cotreatment with CASP3siRNA injected via the tail vein in a 48-h mouse renal IR model. To delineate the possible mechanisms of single/simultaneous administration, the modern technology of transcriptomic microarray analysis was also used to disclose a transcriptional overview in an array of genes and their biological involvements.

\section{Materials And Methods}

\section{CHBP}

The sequence of CHBP was the same with HBSP, QEQLERALNSS, and it was thioether-cyclized (molecule weight 1416.8). CHBP was synthesized by Shanghai Institute of Materia Medica, Chinese Academy of Sciences, China.

\section{Caspase-3 siRNA}

CASP3siRNA, targeting murine caspase-3 mRNA (NCBI CoreNucleotide Accession No. BC038825), were synthesized (Life Technologies, Paisley, UK). The sequences of the CASP3siRNA (Ambion In Vivo, catalogue number: 4457309) were sense 5'-CCUGGUUACUAUUCCUGGAtt-3' and antisense 5'UCCAGGAAUAGUAACCAG

Gtg-3'. The negative control siRNA (NCsiRNA) was also provided by Life Technologies (Ambion In Vivo, catalogue number: 4457289), with sequences of sense 5'-UAACGACGCGACGACGUAAtt-3' and antisense 5'-UUACGUCGUCGCGU 
CGUUAtt-3'. Both CASP3siRNA and NCsiRNA were chemically modified by locked nuclei acid.

\section{Renal IR surgery}

Male C57BL/6 mice, 8-12 weeks, were purchased from the Experimental Animal Center of Yangzhou University, China. All animal experiments were performed according to the guidelines of the Laboratory Animal Monitoring Committee of Jiangsu Province.

The renal IR surgical procedures were performed under general anesthesia by intraperitoneal (i.p.) injection of pentobarbital sodium at $75 \mathrm{mg} / \mathrm{kg}$ body weight (BW). Bilateral kidneys were exposed via dorsal incisions sequentially, and the renal pedicle was carefully isolated and occluded using a nontraumatic vascular clamp for $30 \mathrm{~min}$. The efficacy of occlusion was confirmed by the color change of kidney surface and to dark red eventually. Followed by removing the clamps, patched blanching appeared to the kidney surface and then normal pink, indicating blood reperfusion. Sham operation was performed in the similar manner, except clamping of renal pedicles. Mice were randomly divided into 7 groups $(n=6$ in each group): (1) Sham; (2) IR; (3) IR + CASP3siRNA; (4) IR + NCsiRNA; (5) IR + CHBP; (6) IR + CHBP + CASP3siRNA; (7) IR + CHBP + NCsiRNA. The experimental design is shown in Fig. 1a. $0.03 \mathrm{mg} / \mathrm{kg}$ BW of siRNA was injected into the tail vein $2 \mathrm{~h}$ pre-surgery. $24 \mathrm{nmol} / \mathrm{kg}$ BW of CHBP was given through i.p. at 15 min after clamps were released.

\section{Sample collection}

At $48 \mathrm{~h}$ of renal IR injury, animals were anaesthetized with pentobarbital sodium, followed by cardiac puncture for drawing whole blood. Serum samples were then obtained by centrifuging at 10,000 rpm for 15 min. Kidneys were removed and transversally cut at the midplane, following crosscutting from the middle. One quarter of each kidney was fixed in $10 \%$ neutral formalin for $24 \mathrm{~h}$, while two quarters were rapidly frozen in liquid nitrogen and the fourth part was preserved in RNAlater (Life Technologies).

\section{Biochemistry analysis}

Serum creatinine (SCr) level was determined using a QuantiChrom ${ }^{\mathrm{TM}}$ Creatinine Assay Kit (BioAssay Systems, Hayward, USA). Briefly, thirty $\mu$ l of standard or sample serum were transferred into a 96-well plate followed by adding in $200 \mu \mathrm{l}$ working reagent per well, a mixture of reagent $A$ and $B$. Absorbance at $510 \mathrm{~nm}$ was read immediately and 5 min later. Calculation was performed according to the manufacturer's instruction.

\section{Histological assessment}

Hematoxylin \& eosin (H\&E) staining of kidney tissues was performed to observe and evaluate the degree of tubulointerstitial damage (TID) in the cortex using a scoring system by assessing tubular damage (degeneration and detachment from basement membrane), interstitial expansion (edema or inflammatory cell infiltration), and dilation of tubular lumina. Histological changes were graded based on the percentage of damaged area involved: $<5 \%$ area was scored $0 ; 5 \%-25 \%$ area was scored $1 ; 25 \%-50 \%$ 
area was scored $2 ; 50 \%-75 \%$ area was scored 3 ; and area exceeding $75 \%$ was scored 4 . Kidney sections were blindly reviewed by two researchers independently. The scores from three compartments (tubular and interstitial areas, tubular lumina) of each kidney were obtained from 12 fields at 200 magnifications. The average scores per field of three compartments were then summed up for each kidney. The final score of animal was then calculated by averaging the scores from left and right kidneys.

\section{In Situ End-Labeling (ISEL) of apoptotic cells}

Apoptotic cells were detected using a TUNEL Apoptosis Detection Kit (Millipore, MA, USA) by ISEL, as previously described [16]. Paraffin-embedded kidney sections were de-waxed and digested by proteinase $\mathrm{K}$ at $20 \mu \mathrm{g} / \mathrm{ml}$ for $10 \mathrm{~min}$ at $37^{\circ} \mathrm{C}$. The sections were then applied with equilibration buffer, terminal deoxynucleotidyl transferase (TdT) and anti-digoxigenin-peroxidase sequentially. The labeling of apoptotic cells was then revealed with 3-amino-9-ethylcarbazole (AEC, dark red color). Apoptotic cells were examined at 400 magnifications in up to 20 fields of tubulointerstitial areas in the cortex. The number of positively stained cells in each animal was calculated by averaging the average number per field from left and right kidneys. This was blindly reviewed by two researchers independently.

\section{Immunostaining of active caspase- 3 in kidneys}

Active $17 \mathrm{kDa}$ subunit of caspase-3 was stained on kidney paraffin sections using the method described before [25]. Briefly, sections were de-waxed and performed antigen retrieval before incubation with a rabbit-anti-mouse 17 kDa caspase-3 antibody (1:100 dilution, R\&D System, Abingdon, United Kingdom). For negative control, normal rabbit immunoglobulin $\mathrm{G}$ was applied at the same concentration of primary antibody. $17 \mathrm{kDa}$ caspase-3+ cells were counted at 400 magnifications in up to 20 cortical fields of each kidney by two researchers independently. The number of apoptotic cells for each animal was obtained by averaging the numbers from all fields in both kidneys.

\section{Western blot analysis}

Twenty-five $\mu \mathrm{g}$ of kidney homogenate was separated in reduced SDS-PAGE (sodium dodecyl sulfatepolyacrylamide gel electrophoresis) gels and electroblotted onto a PVDF membrane. The membrane was then blocked in $5 \%$ (weight/volume) non-fat milk, following by probing with an anti-full length caspase-3 antibody (CST, Danvers, USA) at 1: 400 dilution, an anti-high mobility group box 1 (HMGB1) antibody (CST) at 1:1000 or an anti- $\beta$-actin antibody (Abcam, Cambridge, UK) at 1:8000 dilution for overnight at 4 ${ }^{\circ} \mathrm{C}$. The corresponding secondary antibody (Jackson ImmunoResearch Laboratories, West Grove, USA) was then applied to the membrane for $2 \mathrm{~h}$ at room temperature. Afterwards, antibody binding was revealed using ECL substrate (Thermo Scientific, Waltham, Massachusetts, USA) and a Molecular Imager Chemi Doc XRS+system (Bio-Rad, Berkeley, USA).

\section{Microarray analysis}


The kidney stored in RNAlater was performed microarray analysis to reveal the profile of whole genomic transcripts by Shanghai Biotechnology Corporation, China. The detection was done in 4 groups $(n=3$ in each group): IR, IR + CHBP, IR + CHBP + CASP3siRNA and IR + CHBP + NCsiRNA. The RNA integrity and quantity were monitored by the 2100 bioanalyzer (Agilent Technologies, Santa Clara, CA) and NanoDrop One (Thermo Scientific), respectively. Two $\mu$ g RNA with an Integrity Number of no less than 8 was required for the genomic profile analysis. The Agilent Whole Mouse Genome Oligo Microarray was applied to interrogate about 41,174 transcripts targeting 34,000 well-established annotated genes. The criteria of fold change (FC) $>1.414$ (up-regulated genes) or $\mathrm{FC}<-1.414$ (down-regulated genes) and $P<$ 0.05 was used for sorting significant differentially expressed genes (DEGs). The cutoff value of FC was based on the fact that 0.5 cycle was the minimum number of polymerase chain reaction (PCR) cycle to distinguish the expressional differences between two samples.

\section{Validation of candidate DEGs by quantitative PCR (qPCR)}

Total RNAs were extracted by Trizol reagent from the kidney tissues of the same animals selected for microarray analysis. One $\mu \mathrm{g}$ total RNA was used for reverse transcription in a $20 \mu \mathrm{l}$ reaction system supplemented with $4 \mu \mathrm{l} 5 \mathrm{x}$ HiScript II qRT SuperMix and RNase-free water using a kit of HiScript II Q RT SuperMix for qPCR (Vazyme, Nanjing, China). The temperature setting was $50^{\circ} \mathrm{C} 15 \mathrm{~min}$, followed by $85^{\circ} \mathrm{C} 2 \mathrm{~min}$. One $\mu \mathrm{l}$ of cDNA product was amplified within a SYBR reaction system (Bioline, London, UK) containing $200 \mathrm{nM}$ forward and reverse primers (Table 1, Biomics, Nantong, China) at $95^{\circ} \mathrm{C}$ for $10 \mathrm{~min}$ followed by 40 cycles of $95^{\circ} \mathrm{C}$ for $15 \mathrm{~s}$ and $55^{\circ} \mathrm{C}$ for $60 \mathrm{~s}$. The level of $\beta$-actin mRNA was used as an endogenous control.

\section{Gene function analysis}

Functional enrichment analysis of significant DEGs identified between groups was performed using Gene Ontology (GO, http://geneontology.org/) [26]. The resulting GO terms with $P$ value less than 0.05 were considered significantly enriched.

\section{Statistical analysis}

Data was expressed as mean \pm standard error of the mean (SEM). Statistical analysis of the data was performed using GraphPad Prism v8.0 software. One-way ANOVA analysis was used to check the homogeneity of variance for more than two groups. Unpaired student TTEST was then carried out to compare between parameters from two groups. $P$ value $<0.05$ was considered as statistically significant.

\section{Results}

\section{Improved kidney function and structure}

At $48 \mathrm{~h}$, the SCr level raised by IR was significantly reduced by the treatment of CHBP, CASP3siRNA or CHBP + CASP3siRNA (Fig. 1b). However, no significant difference was observed among these treatments. 
IR mice treated with CASP3siRNA or CHBP + CASP3siRNA demonstrated a significant lower SCr level than those treated with NCsiRNA or CHBP + NCsiRNA, respectively.

CHBP, CASP3siRNA and CHBP+CASP3siRNA treatment significantly decreased the extent of TID in IR kidneys (Fig. 1C, d). Furthermore, IR mice with co-treatment of CHBP and CASP3siRNA exhibited a lower level of TID in contrasted to those treated with CHBP only or CASP3siRNA only ( $1.43 \pm 0.15$ versus $2.72 \pm$ 0.09 or $2.49 \pm 0.13, P<0.01$ ). In addition, CASP3siRNA-treated IR or IR+CHBP mice demonstrated a significant decrease of TID compared with corresponding NCsiRNA controls.

\section{Alleviated apoptosis}

As anti-apoptosis is a shared renoprotective effect used by CHBP and CASP3siRNA, immunolabelling was performed to examine their effectiveness on reducing kidney IR-induced apoptosis. IR significantly raised the number of ISEL+ cells compared with sham controls, but greatly lowered by CHBP, CASP3siRNA and CHBP + CASP3siRNA (Fig. 2a, b). Nevertheless, comparable levels of ISEL+ cells were found among these treatments. In contrast to NCsiRNA controls, CASP3siRNA reduced apoptosis in either IR kidneys or CHBP-modified IR kidneys.

\section{Decreased active caspase-3 staining positive cells}

Cells labeled with active $17 \mathrm{kDa}$ caspase- 3 often having the morphological features of apoptosis as condensed nuclei were mainly located in tubular epithelia, tubular lumina and interstitial areas. The number of active caspase-3+ cells was significantly increased by IR, but decreased by CHBP, CASP3siRNA and CHBP + CASP3siRNA (Fig. 3a, b). No significant differences between these treatments were observed. Comparing with NCsiRNA, CASP3siRNA significantly reduced the number of $17 \mathrm{kDa}$ caspase$3+$ cells in IR kidneys, as well as in IR + CHBP kidneys.

\section{Decreased expression of active caspase-3 and HMGB1 protein}

Western blotting was used to determine whether there is a co-effect of CHBP and CASP3siRNA on the expression of $17 \mathrm{kDa}$ active caspase-3. The level of $17 \mathrm{kDa}$ caspase-3 was significantly increased by IR, but reduced by CHBP, CASP3siRNA and CHBP + CASP3siRNA (Fig. 4a, b). IR mice with the co-treatment of CHBP and CASP3siRNA showed an even lower expression of $17 \mathrm{kDa}$ caspase- 3 compared with IR mice treated with CASP3siRNA $(0.29 \pm 0.03$ versus $0.52 \pm 0.07, P<0.05)$. Controlled by NCsiRNA, $17 \mathrm{kDa}$ caspase- 3 was reduced by CASP3siRNA in IR kidneys or IR + CHBP kidneys.

HMGB1 is a proinflammatory factor known to arouse profound innate responses by binding to toll-like receptor 4 on surface of tubular epithelial cells (TECs) and macrophages [27, 28]. Western blotting using kidney homogenates demonstrated IR significantly increased HMGB1 expression compared with sham controls (Fig. 4c, d). However, treatments with CHBP, CASP3siRNA and CHBP + CASP3siRNA decreased the high level of HMGB1. Moreover, IR mice with co-treatment of CHBP and CASP3siRNA exhibited an even lower level of renal HMGB1 compared with CASP3siRNA-treated animals $(0.81 \pm 0.16$ versus $1.57 \pm$ 
0.24, $P<0.05)$. In contrast to NCsiRNA, CASP3siRNA reduced HMGB-1 expression in IR kidneys as well as in IR + CHBP kidneys.

\section{Identification of differentially expressed genes and re-validation}

To disclose the mechanism of renoprotection induced by CHBP and/or CASP3siRNA, transcriptomic microarray analysis was conducted to identify DEGs affected in the IR kidneys. 281 DEGs (153 upregulated, 128 down-regulated) were identified in the CHBP treated IR kidneys versus IR kidneys (Fig. 5a). 418 DEGs (226 up-regulated, 192 down-regulated) were shown by the additional administration of CASP3siRNA to CHBP-treated IR kidneys versus IR+CHBP kidneys $₫$ with 46 genes in common to the comparison of IR+CHBP versus IR groups. In contrast to the NCsiRNA treatment to IR+CHBP kidneys, CASP3siRNA produced 504 DEGs (218 up-regulated, 286 down-regulated) in IR+CHBP kidneys, of which 9 genes were commonly altered with the IR+CHBP kidneys versus IR kidneys, and 37 genes in common with the comparison of IR+CHBP+CASP3siRNA versus IR+CHBP. Among above three comparisons, there were only 3 genes affected universally. The top 5 genes of up-regulated and down-regulated in three comparisons were listed (Table 2-4). Among DEGs, up-regulated BCL6 was associated with the negative regulation of apoptosis (Table 2), up-regulated SLPI and SERPINA3M were related to inflammation (Table 3), and up-regulated GREM1 and down-regulated ANGPTL2 linked to injury, inflammation and microvascular homeostasis (Table 4).

To validate the outcome of microarray analysis, 4 DEGs were selected for qPCR detection: up-regulated SLC22A7 by CHBP compared with the IR group (FC = 2.996), associated with the epithelial function of organic anion transport; up-regulated CFH by CASP3siRNA compared with NCsiRNA (FC = 1.949), a negative regulator in the alternative pathway of complement activation; and ANGPTL2 and GREM1 as described above. QPCR results showed that the level of SLC22A7 was greatly up-regulated by CHBP (Fig. 5b), so were CFH and GREM1 increased (Fig. 5c, d), but Angptl2 was decreased by CASP3siRNA compared with NCsiRNA (Fig. 5e). Thus, all results from qPCR were consistent with the output of microarray data.

\section{GO analysis of the DEGs}

The identified DEGs were subjected to GO functional enrichment analysis to elucidate biological processes altered by CHBP and/or CASP3siRNA in the IR kidneys at $48 \mathrm{~h}$. Top 30 items of biological process $(P<0.05)$ with enrich factors are presented (Fig. 6a-C). DEGs induced by CHBP were mainly involved in cell division and function (Fig. 6a), for instance, up-regulated signal transducer and activator of transcription 5B (STAT5B, FC = 1.478), a positive regulator of mitotic cell cycle; up-regulated SLC22A7, mediating organic anion transport, as well as positive regulator of cellular component biogenesis and glucose metabolic process. Further altered genes by CASP3siRNA treatment in IR + CHBP kidneys versus $\mathrm{IR}+\mathrm{CHBP}$ involved in the negative regulation of immune response (Fig. 6b). Compared with the NCsiRNA control, CASP3siRNA further affected inflammatory and cell death signaling in IR+CHBP kidneys, such as regulation of interleukin-1 beta (IL-1 $\beta$ ) production, positive regulation of phosphatidylinositol 3-kinase signaling, release of cytochrome c from mitochondria (Fig. 6c). 


\section{Discussion}

The present study demonstrated that a single dose of CHBP or CASP3siRNA markedly ameliorated IRinduced kidney injury in terms of preserving renal function and structure, reducing active caspase- 3 and HMGB1 expression. The combination of both further decreased TID, active caspase- 3 and HMGB1. In addition, genomic microarray analysis identified DEGs induced by CHBP were mainly involved in preserving cell division, cellular function and metabolism. DEGs modified by CASP3siRNA were associated with inhibiting inflammation and maintaining vascular function. Certain genes such as BCL6, SLPI, SERPINA3M, GREM1 and ANGPTL2 might be potential biomarkers in IR-induced AKI.

The present study demonstrated that a single dose of CHBP (plasma half-life 300 min [13]) administrated 15 min after reperfusion greatly ameliorated renal IR injury at the early stage of $48 \mathrm{~h}$. This result was consistent with the evidence that a single dose of CHBP protected the kidney from IR injury at 12-week [20]. Linear HBSP (plasma half-life about $2 \mathrm{~min}$ ) administered at $1 \mathrm{~h}, 6 \mathrm{~h}$ and $12 \mathrm{~h}$ protected the kidney against IR injury at $24 \mathrm{~h}$ [14]. Our previous study also showed that daily injection of HBSP protected the kidney from immunosuppressant cyclosporine A-induced damage upon IR injury, but did not affect IR injury alone in a 2-w rat model [16]. It has been also reported that CHBP protected against aristolochic acid induced AKI [29]. These data imply a variety of potential clinical applications of CHBP or HBSP.

It is the first time verifying that a single dose of CASP3siRNA was comparable to CHBP in renal protection. siRNA is a potent and specific tool that can silence detrimental genes under disease conditions $₫$ so siRNA therapy provides prospective in the development of precision medicine [30]. Although there are over 30 siRNA-related clinical trials that have been completed, no siRNA treatment against AKI is available in clinical practice. The result from this study implies that caspase- 3 gene may be one of major affected genes by CHBP in renoprotection, therefore, CASP3siRNA might be an alternative treatment additional to CHBP for IR-induced renal injury.

The transcriptomic profile, moreover, demonstrated that CHBP altered genes in biological processes were mainly linked to cell division $₫$ cellular function and metabolism. For example, STAT5B up-regulated by CHBP was involved in cell proliferation in rodent kidneys [31, 32], while SLC22A7, enriched in organic anion transport, was associated with the extrusion of creatinine from TECs and maintaining SCr level [33]. BCL6, among the top 5 DEGs up-regulated by CHBP (Table 2), has a broad role on anti-apoptosis and cell survival [34], promoting the expression of organic anion transporter 1 in TECs and maintaining the secreting function of TECs [35]. In addition, metabolic processes were greatly enriched by CHBP including glucose metabolism, which is beneficial for energy production [36]. It has also been reported that the proteome profile in IR kidneys at $48 \mathrm{~h}$ changed by CHBP treatment was mainly related to the oxidative stress [37]. There may be differentiations between transcriptional and translational changes, as well as the stain of mouse strain (BALB/c) and dose of CHBP $(8 \mathrm{nmol} / \mathrm{kg})$.

Intriguingly, in contrast to single CHBP or CASP3siRNA treatment, co-treatment with CHBP and CASP3siRNA contributed to further preservation in renal structure, with lower active caspase-3 and HMGB1 in IR kidneys. The negative regulation of immune responses was also revealed by microarray 
analysis, verifying the effectiveness of further CASP3siRNA against renal IR. SLPI, secretory leukocyte peptidase inhibitor》 among the top 5 DEGs up-regulated by CASP3 siRNA (Table 3), was renoprotective in experimental ischemia AKI [38]. SLPI inhibits nuclear factor kappa beta (NF-KB) signaling pathway [39] and the maturation of IL-1 [40], and shown as a biomarker candidate in AKI [41, 42]. In human, SERPINA3, a member of the serpin superfamily of protease inhibitors, could limit inflammation by targeting cathepsin family (proinflammatory enzymes) [43, 44]. SERPINA3 expression was also found in rat kidneys, which can detect renal inflammation and fibrosis after IR injury and also serve as a urinary marker for early detection of AKI to CKD transition [45]. Because murine SERPINA3M (FC $=8.289$, Table 3 ) is a likely orthologue of human SERPINA3, the two proteins may have similar structural and kinetical characterization. The role of SERPINA3M in renal IR injury is worthy of further exploring.

Similar effects of renoprotection from CASP3siRNA were also revealed by comparing with NCsiRNA control in CHBP-treated IR kidneys. Microarray data revealed that further CASP3siRNA treatment significantly regulated renal inflammation and programmed cell death upon CHBP compared with that of NCsiRNA. CFH, a negative regulator of complement alternative pathway that plays crucial roles in IR injury [46], was increased by further CASP3siRNA treatment. Notably, ANGPTL2 was the top one of downregulated DEGs by CASP3siRNA (Table 4). Less Angpt12 could contribute to the reduction of renal inflammation as ANGPTL2 can activate resident macrophages and induce the secretion of proinflammatory cytokines $[47,48]$. Decreased ANGPTL2 may also ameliorate renal fibrosis in AKIinduced chronic kidney disease by depressing transforming growth factor- $\beta$ (TGF- $\beta$ ) signaling [49]. In addition, GREM1 was the top one of up-regulated DEGs by CASP3siRNA (Table 4), which activates vascular endothelial growth factor receptor 2 (VEGFR2) in endothelial cells to induce angiogenesis [50, 51]. The effective repair of endothelial cells in IR-injured kidney plays essential roles in maintaining the homeostasis of microvasculature and efficient renal blood flow [52], and subsequently ameliorating tubular damage [53]. The GREM1-VEGFR2 axis may be a novel therapeutic target for kidney inflammation and fibrosis [54]. In addition, Yang and colleagues proposed that caspase-3 deficiency in mice reduced IR injury in kidneys through preserving microvascular density [55]. However, whether the preservation of renal microvasculature in this study links to regulated GREM1 is worthy of further investigating. The above evidence indicates a promising strategy of silencing caspase- 3 and administrating CHBP at the same time for optimized outcome in improving IR injury in kidneys.

Special attention should be paid to the toxicity of NCsiRNA in the present study, which was evidenced by further elevated SCr, TID and apoptotic levels in CHBP-modified IR kidneys. These data suggested that the synthetic siRNA duplexes may still modulate immunity and inflammation in IR kidneys, such as releasing cytokines and interferons, and activating toll-like receptors on immune and nonimmune cells [56-58]. It is indicating that NCsiRNA might down-regulate the influence of CHBP treatment on IR kidneys, providing an ideal and necessary control for the specific effects of CASP3siRNA in the context of CHBP-treated IR kidneys.

There are also limitations in this study. The additive renoprotective effect of CASP3siRNA on CHBP should be further studied in the long-term prognosis of IR injury. In addition, DEGs identified by the 
present microarray analysis should be further validated in more comparisons with the groups including more samples. Moreover, to select and validate potential biomarkers from identified DEGs, different downstream biological events at translational and post-translational level should be further investigated in terms of its dynamic expression, regulation and intervention.

\section{Conclusions}

The co-treatment of CHBP and CASP3siRNA exhibited synergistic effects on renoprotection against 48-h renal IR in a mouse model. The DEGs induced by CHBP are associated with the preservation of cell division, function and metabolism, while the DEGs caused by CASP3siRNA are linked to improving inflammation and potential microvasculature.

\section{List Of Abbreviations}

AEC: 3-amino-9-ethylcarbazole; AKI: Acute kidney injury; Angptl2: Angiopoietin-like 2; Bcl6: B cell leukemia/lymphoma 6; BW: Body weight; CASP3siRNA: Caspase-3 small interfering RNA; CFH:

Complement factor $\mathrm{H}$; CHBP: Cyclic helix B surface peptide; DEGs: Differentially expressed genes; EPO: Erythropoietin; EPOR/ $\beta c R$ : Erythropoietin receptor and $\beta$ common receptor; FC: Fold change; FoxO3a: Forkhead box 0 3a; GO: Gene Ontology; GREM1: Gremlin 1; H\&E: Hematoxylin \& eosin; HBSP: Helix B surface peptide; HMGB1: High mobility group box 1; IR: Ischemia/reperfusion; ISEL: In situ end-labeling; NCsiRNA: Negative control small interfering RNA; qPCR: Quantitative polymerase chain reaction; SCr: Serum creatinine; SDS-PAGE: Sodium dodecyl sulfate-polyacrylamide gel electrophoresis; SEM: Standard error of the mean; siRNA: Small interfering RNA; SLC22A7: Solute carrier family 22 member 7; SLPI: Secretory leukocyte peptidase inhibitor; STAT5B: Signal transducer and activator of transcription 5B; TdT: Terminal deoxynucleotidyl transferase; TECs: Tubular epithelial cells; TID: Tubulointerstitial damage; VEGFR2: Vascular endothelial growth factor receptor 2.

\section{Declarations}

\section{Ethic approval and consent to participate}

Human subjects: not applicable. Animals: This study was approved by the Laboratory Animal Monitoring Committee of Jiangsu Province with established guidelines for the care and use of laboratory animals.

\section{Consent for publication}

Not applicable.

\section{Availability of data materials}

All data generated or analyzed during this study are included in this published article.

\section{Competing interests:}


There is no financial conflict in the information contained in this manuscript.

\section{Finding}

This study was supported by the National Nature Science Foundation of China (Foundation Numbers: 81170689,81570677 and 81873622 to Bin Yang). Other support was provided from the University of Nantong and the Research \& Development Directorate of the University Hospitals of Leicester NHS Trust.

\section{Authors' contributions}

BY conceived and designed the study. YW, WC, CY established the animal models, performed biochemical and molecular biological detection. $\mathrm{YZ}, \mathrm{AL}$ and $\mathrm{HW}$ performed histological staining and assessment. $\mathrm{YW}$ collected the data, performed data analysis and interpretation, as well as wrote the manuscript. TZ, YF and BY revised the manuscript. All authors read and approved the final manuscript.

\section{Acknowledgments}

We would like to acknowledge the Comparative Medicine Institute of Nantong University for providing all facilities to accommodate this study, and Professor Yaqiu Long (Key Laboratory of Receptor Research, Shanghai Institute of Materia Medica, Chinese Academy of Sciences) for providing CHBP for this study.

\section{References}

1. Mehta RL, Burdmann EA, Cerda J, Feehally J, Finkelstein F, Garcia-Garcia G, et al. Recognition and management of acute kidney injury in the International Society of Nephrology 0by 25 Global Snapshot: a multinational cross-sectional study. Lancet. 2016;387:2017-25.

2. Yang $L$, Xing G, Wang L, Wu Y, Li S, Xu G, et al. Acute kidney injury in China: a cross-sectional survey. Lancet. 2015;386:1465-71.

3. Bouchard J, Acharya A, Cerda J, Maccariello ER, Madarasu RC, Tolwani AJ, et al. A Prospective International Multicenter Study of AKI in the Intensive Care Unit. Clin J Am Soc Nephrol. 2015;10:1324-31.

4. Moore PK, Hsu RK, Liu KD. Management of Acute Kidney Injury: Core Curriculum 2018. Am J Kidney Dis. 2018;72:136-48.

5. Mehta RL, Cerda J, Burdmann EA, Tonelli M, Garcia-Garcia G, Jha V, et al. International Society of Nephrology's 0by25 initiative for acute kidney injury (zero preventable deaths by 2025): a human rights case for nephrology. Lancet. 2015;385:2616-43.

6. Noble RA, Lucas BJ, Selby NM. Long-Term Outcomes in Patients with Acute Kidney Injury. Clin J Am Soc Nephrol. 2020;15:423-9.

7. Dong Y, Zhang Q, Wen J, Chen T, He L, Wang Y, et al. Ischemic Duration and Frequency Determines AKI-to-CKD Progression Monitored by Dynamic Changes of Tubular Biomarkers in IRI Mice. Front Physiol. 2019;10:153. 
8. Bellomo R, Kellum JA, Ronco C. Acute kidney injury. Lancet. 2012;380:756-66.

9. Brines M, Cerami A. The receptor that tames the innate immune response. Mol Med. 2012;18:486-96.

10. Wang S, Zhang C, Li J, Niyazi S, Zheng L, Xu M, et al. Erythropoietin protects against rhabdomyolysis-induced acute kidney injury by modulating macrophage polarization. Cell Death Dis. 2017;8:e2725.

11. Gobe GC, Bennett NC, West M, Colditz P, Brown L, Vesey DA, et al. Increased progression to kidney fibrosis after erythropoietin is used as a treatment for acute kidney injury. Am J Physiol Renal Physiol. 2014;306:F681-92.

12. Shi M, Flores B, Li P, Gillings N, McMillan KL, Ye J, et al. Effects of erythropoietin receptor activity on angiogenesis, tubular injury, and fibrosis in acute kidney injury: a "U-shaped" relationship. Am J Physiol Renal Physiol. 2018;314:F501-F16.

13. Yang C, Xu Z, Zhao Z, Li L, Zhao T, Peng D, et al. A novel proteolysis-resistant cyclic helix B peptide ameliorates kidney ischemia reperfusion injury. Biochim Biophys Acta. 2014;1842:2306-17.

14. Brines M, Patel NS, Villa P, Brines C, Mennini T, De Paola M, et al. Nonerythropoietic, tissue-protective peptides derived from the tertiary structure of erythropoietin. Proc Natl Acad Sci U S A. 2008;105:10925-30.

15. Patel NS, Kerr-Peterson HL, Brines M, Collino M, Rogazzo M, Fantozzi R, et al. Delayed administration of pyroglutamate helix B surface peptide (pHBSP), a novel nonerythropoietic analog of erythropoietin, attenuates acute kidney injury. Mol Med. 2012;18:719-27.

16. Wu Y, Zhang J, Liu F, Yang C, Zhang Y, Liu A, et al. Protective effects of HBSP on ischemia reperfusion and cyclosporine a induced renal injury. Clin Dev Immunol. 2013;2013:758159.

17. Yang C, Zhao T, Lin M, Zhao Z, Hu L, Jia Y, et al. Helix B surface peptide administered after insult of ischemia reperfusion improved renal function, structure and apoptosis through beta common receptor/erythropoietin receptor and PI3K/Akt pathway in a murine model. Exp Biol Med (Maywood). 2013;238:111-9.

18. Zhang Y, Wang Q, Liu A, Wu Y, Liu F, Wang H, et al. Erythropoietin Derived Peptide Improved Endoplasmic Reticulum Stress and Ischemia-Reperfusion Related Cellular and Renal Injury. Front Med (Lausanne). 2020;7:5.

19. Kaushal GP, Shah SV. Autophagy in acute kidney injury. Kidney Int. 2016;89:779-91.

20. Yang $C$, Cao Y, Zhang Y, Li L, Xu M, Long Y, et al. Cyclic helix B peptide inhibits ischemia reperfusioninduced renal fibrosis via the PI3K/Akt/FoxO3a pathway. J Transl Med. 2015;13:355.

21. Li Y, Hou D, Chen X, Zhu J, Zhang R, Sun W, et al. Hydralazine protects against renal ischemiareperfusion injury in rats. Eur J Pharmacol. 2019;843:199-209.

22. Yang B, Hosgood SA, Bagul A, Waller HL, Nicholson ML. Erythropoietin regulates apoptosis, $i$ nflammation and tissue remodelling via caspase-3 and IL-1 beta in isolated hemoperfused kidneys. Eur J Pharmacol. 2011;660:420-30. 
23. Yang C, Hosgood SA, Meeta P, Long Y, Zhu T, Nicholson ML, et al. Cyclic Helix B Peptide in Preservation Solution and Autologous Blood Perfusate Ameliorates Ischemia-Reperfusion Injury in Isolated Porcine Kidneys. Transplant Direct. 2015;1:e6.

24. Yang C, Zhao T, Zhao Z, Jia Y, Li L, Zhang Y, et al. Serum-stabilized naked caspase-3 siRNA protects autotransplant kidneys in a porcine model. Mol Ther. 2014;22:1817-28.

25. Yang B, Hosgood SA, Nicholson ML. Naked small interfering RNA of caspase-3 in preservation solution and autologous blood perfusate protects isolated ischemic porcine kidneys. Transplantation. 2011;91:501-7.

26. Harris MA, Clark J, Ireland A, Lomax J, Ashburner M, Foulger R, et al. The Gene Ontology (GO) database and informatics resource. Nucleic Acids Res. 2004;32:D258-61.

27. Chen CB, Liu LS, Zhou J, Wang XP, Han M, Jiao XY, et al. Up-Regulation of HMGB1 Exacerbates Renal Ischemia-Reperfusion Injury by Stimulating Inflammatory and Immune Responses through the TLR4 Signaling Pathway in Mice. Cell Physiol Biochem. 2017;41:2447-60.

28. Wu H, Ma J, Wang P, Corpuz TM, Panchapakesan U, Wyburn KR, et al. HMGB1 contributes to kidney ischemia reperfusion injury. J Am Soc Nephrol. 2010;21:1878-90.

29. Zeng Y, Zheng L, Yang Z, Yang C, Zhang Y, Li J, et al. Protective effects of cyclic helix B peptide on aristolochic acid induced acute kidney injury. Biomed Pharmacother. 2017;94:1167-75.

30. Hawgood S, Hook-Barnard IG, O'Brien TC, Yamamoto KR. Precision medicine: Beyond the inflection point. Sci Transl Med. 2015;7:300ps17.

31. Chen SC, Guh JY, Chen HC, Yang YL, Huang JS, Chuang LY. Advanced glycation end-product-induced mitogenesis is dependent on Janus kinase 2-induced heat shock protein 70 in normal rat kidney interstitial fibroblast cells. Transl Res. 2007;149:274-81.

32. Fragiadaki M, Lannoy M, Themanns M, Maurer B, Leonhard WN, Peters DJ, et al. STAT5 drives abnormal proliferation in autosomal dominant polycystic kidney disease. Kidney Int. 2017;91:575-86.

33. Shen H, Liu T, Morse BL, Zhao Y, Zhang Y, Qiu X, et al. Characterization of Organic Anion Transporter 2 (SLC22A7): A Highly Efficient Transporter for Creatinine and Species-Dependent Renal Tubular Expression. Drug Metab Dispos. 2015;43:984-93.

34. Baron BW, Hyjek E, Gladstone B, Thirman MJ, Baron JM. PDCD2, a protein whose expression is repressed by BCL6, induces apoptosis in human cells by activation of the caspase cascade. Blood Cells Mol Dis. 2010;45:169-75.

35. Wegner W, Burckhardt G, Henjakovic M. Transcriptional regulation of human organic anion transporter 1 by B-cell CLL/lymphoma 6. Am J Physiol Renal Physiol. 2014;307:F1283-91.

36. Wei Q, Xiao X, Fogle P, Dong Z. Changes in metabolic profiles during acute kidney injury and recovery following ischemia/reperfusion. PLoS One. 2014;9:e106647.

37. Yang C, Liu J, Li L, Hu M, Long Y, Liu X, et al. Proteome Analysis of Renoprotection Mediated by a Novel Cyclic Helix B Peptide in Acute Kidney Injury. Sci Rep. 2015;5:18045. 
38. Ochi A, Chen D, Schulte W, Leng L, Moeckel N, Piecychna M, et al. MIF-2/D-DT enhances proximal tubular cell regeneration through SLPI- and ATF4-dependent mechanisms. Am J Physiol Renal Physiol. 2017;313:F767-F80.

39. Tang R, Botchway BOA, Meng Y, Zhang Y, Zhou C, Jiang J, et al. The Inhibition of Inflammatory Signaling Pathway by Secretory Leukocyte Protease Inhibitor can Improve Spinal Cord Injury. Cell Mol Neurobiol. 2020.

40. Zakrzewicz A, Richter K, Zakrzewicz D, Siebers K, Damm J, Agne A, et al. SLPI Inhibits ATP-Mediated Maturation of IL-1beta in Human Monocytic Leukocytes: A Novel Function of an Old Player. Front Immunol. 2019;10:664.

41. Averdunk L, Ruckbeil MV, Zarbock A, Martin L, Marx G, Jalaie H, et al. SLPI - a Biomarker of Acute Kidney Injury after Open and Endovascular Thoracoabdominal Aortic Aneurysm (TAAA) Repair. Sci Rep. 2020;10:3453.

42. Averdunk L, Fitzner C, Levkovich T, Leaf DE, Sobotta M, Vieten J, et al. Secretory Leukocyte Protease Inhibitor (SLPI)-A Novel Predictive Biomarker of Acute Kidney Injury after Cardiac Surgery: A Prospective Observational Study. J Clin Med. 2019;8.

43. Lannan EA, Galliher-Beckley AJ, Scoltock AB, Cidlowski JA. Proinflammatory actions of glucocorticoids: glucocorticoids and TNFalpha coregulate gene expression in vitro and in vivo. Endocrinology. 2012;153:3701-12.

44. Horvath AJ, Irving JA, Rossjohn J, Law RH, Bottomley SP, Quinsey NS, et al. The murine orthologue of human antichymotrypsin: a structural paradigm for clade A3 serpins. J Biol Chem. 2005;280:4316878.

45. Sanchez-Navarro A, Mejia-Vilet JM, Perez-Villalva R, Carrillo-Perez DL, Marquina-Castillo B, Gamba G, et al. SerpinA3 in the Early Recognition of Acute Kidney Injury to Chronic Kidney Disease (CKD) transition in the rat and its Potentiality in the Recognition of Patients with CKD. Sci Rep. 2019;9:10350.

46. Goetz L, Laskowski J, Renner B, Pickering MC, Kulik L, Klawitter J, et al. Complement factor H protects mice from ischemic acute kidney injury but is not critical for controlling complement activation by glomerular IgM. Eur J Immunol. 2018;48:791-802.

47. Umikawa M, Umikawa A, Asato T, Takei K, Matsuzaki G, Kariya K, et al. Angiopoietin-like protein 2 induces proinflammatory responses in peritoneal cells. Biochem Biophys Res Commun. 2015;467:235-41.

48. Amadatsu T, Morinaga J, Kawano T, Terada K, Kadomatsu T, Miyata K, et al. Macrophage-Derived Angiopoietin-Like Protein 2 Exacerbates Brain Damage by Accelerating Acute Inflammation after Ischemia-Reperfusion. PLoS One. 2016;11:e0166285.

49. Morinaga J, Kadomatsu T, Miyata K, Endo M, Terada K, Tian Z, et al. Angiopoietin-like protein 2 increases renal fibrosis by accelerating transforming growth factor-beta signaling in chronic kidney disease. Kidney Int. 2016;89:327-41. 
50. Ravelli C, Mitola S, Corsini M, Presta M. Involvement of alphavbeta3 integrin in gremlin-induced angiogenesis. Angiogenesis. 2013;16:235-43.

51. Ji C, Huang JW, Xu QY, Zhang J, Lin MT, Tu Y, et al. Gremlin inhibits UV-induced skin cell damages via activating VEGFR2-Nrf2 signaling. Oncotarget. 2016;7:84748-57.

52. Kwon O, Miller S, Li N, Khan A, Kadry Z, Uemura T. Bone marrow-derived endothelial progenitor cells and endothelial cells may contribute to endothelial repair in the kidney immediately after ischemiareperfusion. J Histochem Cytochem. 2010;58:687-94.

53. Cantaluppi V, Gatti S, Medica D, Figliolini F, Bruno S, Deregibus MC, et al. Microvesicles derived from endothelial progenitor cells protect the kidney from ischemia-reperfusion injury by microRNAdependent reprogramming of resident renal cells. Kidney Int. 2012;82:412-27.

54. Mezzano S, Droguett A, Lavoz C, Krall P, Egido J, Ruiz-Ortega M. Gremlin and renal diseases: ready to jump the fence to clinical utility? Nephrol Dial Transplant. 2018;33:735-41.

55. Yang B, Lan S, Dieude M, Sabo-Vatasescu JP, Karakeussian-Rimbaud A, Turgeon J, et al. Caspase-3 Is a Pivotal Regulator of Microvascular Rarefaction and Renal Fibrosis after Ischemia-Reperfusion Injury. J Am Soc Nephrol. 2018;29:1900-16.

56. Robbins M, Judge A, MacLachlan I. siRNA and innate immunity. Oligonucleotides. 2009;19:89-102.

57. Judge AD, Sood V, Shaw JR, Fang D, McClintock K, MacLachlan I. Sequence-dependent stimulation of the mammalian innate immune response by synthetic siRNA. Nat Biotechnol. 2005;23:457-62.

58. Forsbach A, Nemorin JG, Montino C, Muller C, Samulowitz U, Vicari AP, et al. Identification of RNA sequence motifs stimulating sequence-specific TLR8-dependent immune responses. J Immunol. 2008;180:3729-38.

\section{Tables}

Table 1. The sequence of primers for quantitative polymerase chain reaction (qPCR).

\begin{tabular}{|c|c|c|}
\hline Gene symbol & Full name & Primers $\left(5^{\prime}-3^{\prime}\right)$ \\
\hline SLC22A7 & solute carrier family 22 member 7 & $\begin{array}{l}\text { Forward: CTGTCTGCCTGTGTTTATCC } \\
\text { Reverse: CTTCCCCAAATGCCACAGCT }\end{array}$ \\
\hline $\mathrm{CFH}$ & complement factor $\mathrm{H}$ & $\begin{array}{l}\text { Forward: ACTTTCTCAGATTTTCCTGG } \\
\text { Reverse: TGGTTGTTACATGCTTTGGG }\end{array}$ \\
\hline ANGPTL2 & angiopoietin-like 2 & $\begin{array}{l}\text { Forward: GGATGGTTCACAGAGAGAGTAC } \\
\text { Reverse: CTCCTTGGAGTTGACACAAATG }\end{array}$ \\
\hline GREM1 & gremlin-1 & $\begin{array}{l}\text { Forward: GCAAGTATCTGAAGVGAGATTG } \\
\text { Reverse: CGTCATGGTGGTGAACTTCTTG }\end{array}$ \\
\hline ACTB & $\beta$-actin & $\begin{array}{l}\text { Forward: GAGACCTTCAACACCCCAGC } \\
\text { Reverse: ATGTCACGCACGATTTCCC }\end{array}$ \\
\hline
\end{tabular}

Table 2. IR+CHBP versus IR, top 5 up-regulated and down-regulated. $(P<0.05)$ 


\begin{tabular}{llll}
\hline Accession NO. & Gene symbol & Gene name & Fold change \\
\cline { 1 - 3 } Up-regulated & & & \\
AK016105 & 4930553I04RIK RIKEN cDNA 4930553I04 gene & 8.559 \\
AK046501 & SACS & sacsin & 5.261 \\
NM_013501 & CRYAA & crystallin, alpha A & 4.357 \\
NM_011255 & RBP4 & retinol binding protein 4, plasma & 4.213 \\
NM_009744 & BCL6 & B cell leukemia/lymphoma 6 & 3.622 \\
Down-regulated & & & \\
NM_001167777 & ASXL3 & additional sex combs like 3 & -24.005 \\
NM_029070 & CLDN26 & claudin 26 & -5.985 \\
NM_001167746 & DNAH17 & dynein, axonemal, heavy chain 17 & -5.922 \\
AK171340 & PITPNC1 & phosphatidylinositol transfer protein, cytoplasmic 1 & -5.113 \\
NM_007501 & NEUROD4 & neurogenic differentiation 4 & -5.094 \\
\hline
\end{tabular}

The gene in bold was discussed its function in IR-induced AKI.

Table 3. IR+CHBP+CASP3siRNA versus IR+CHBP, top 5 up-regulated and down-regulated. $(P<0.05)$

\begin{tabular}{|c|c|c|c|}
\hline Accession NO. & Gene symbol & Gene name & Fold change \\
\hline \multicolumn{4}{|l|}{ Up-regulated } \\
\hline NM_001001450 & SSXB2 & $\begin{array}{l}\text { synovial sarcoma, } \mathrm{X} \text { member } \mathrm{B} \text {, } \\
\text { breakpoint } 2\end{array}$ & 14.730 \\
\hline NR_028111 & 4930523C07RIK & RIKEN cDNA 4930523C07 gene & 10.131 \\
\hline NM_011414 & SLPI & secretory leukocyte peptidase inhibitor & 8.478 \\
\hline NM_009253 & SERPINA3M & $\begin{array}{l}\text { serine (or cysteine) peptidase inhibitor, } \\
\text { clade A, member } 3 \mathrm{M}\end{array}$ & 8.289 \\
\hline NM_175309 & UPK3B & uroplakin 3B & 5.483 \\
\hline \multicolumn{4}{|l|}{ Down-regulated } \\
\hline AK016105 & 4930553I04RIK & RIKEN cDNA 4930553104 gene & -8.735 \\
\hline NM_029747 & $2410137 \mathrm{M} 14 \mathrm{RIK}$ & RIKEN cDNA 2410137M14 gene & -5.134 \\
\hline NM_147025 & OLFR380 & olfactory receptor 380 & -4.354 \\
\hline NM_134160 & MCOLN3 & mucolipin 3 & -4.142 \\
\hline NM_001163513 & DLG5 & discs large MAGUK scaffold protein 5 & -3.700 \\
\hline
\end{tabular}

The gene in bold was discussed its function in IR-induced AKI.

Table 4. IR+CHBP+CASP3siRNA versus IR+CHBP+NCsiRNA, top 5 up-regulated and down-regulated $(P<0.05)$ 


\begin{tabular}{|c|c|c|c|}
\hline Accession NO. & \multicolumn{2}{|c|}{ Gene symbol Gene name } & Fold change \\
\hline \multicolumn{4}{|l|}{ Up-regulated } \\
\hline NM_011824 & GREM1 & gremlin 1 & 15.987 \\
\hline NM_001001450 & SSXB2 & $\begin{array}{l}\text { synovial sarcoma, } \mathrm{X} \text { member } \mathrm{B} \text {, } \\
\text { breakpoint } 2\end{array}$ & 14.817 \\
\hline NM_026433 & TMEM100 & transmembrane protein 100 & 9.749 \\
\hline NM_011825 & GREM2 & $\begin{array}{l}\text { gremlin } 2 \text { homolog, cysteine } \\
\text { knot superfamily (Xenopus laevis) }\end{array}$ & 9.312 \\
\hline NM_007729 & COL11A1 & collagen, type XI, alpha 1 & 8.978 \\
\hline \multicolumn{4}{|l|}{ Down-regulated } \\
\hline AK041888 & ANGPTL2 & angiopoietin-like 2 & -24.390 \\
\hline AF1 07847 & GNAS & $\begin{array}{l}\text { GNAS (guanine nucleotide binding } \\
\text { protein, alpha stimulating) complex locus }\end{array}$ & -12.195 \\
\hline AK081320 & ZFYVE9 & $\begin{array}{l}\text { zinc finger, FYVE domain } \\
\text { containing } 9\end{array}$ & -7.576 \\
\hline NM_010258 & GATA6 & GATA binding protein 6 & -6.711 \\
\hline NM_026648 & DNAAF1 & dynein, axonemal assembly factor 1 & -6.579 \\
\hline
\end{tabular}

Genes in bold indicate analysis by quantitative polymerase chain reaction (qPCR) and discussed as biomarker candidates of IR-induced AKI.

\section{Figures}


a

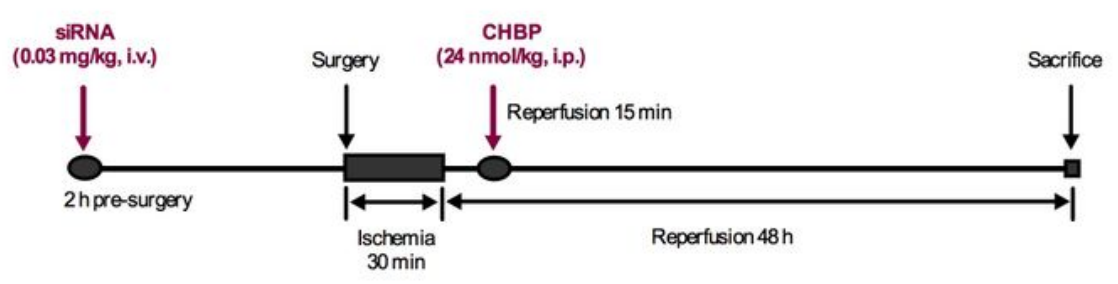

b

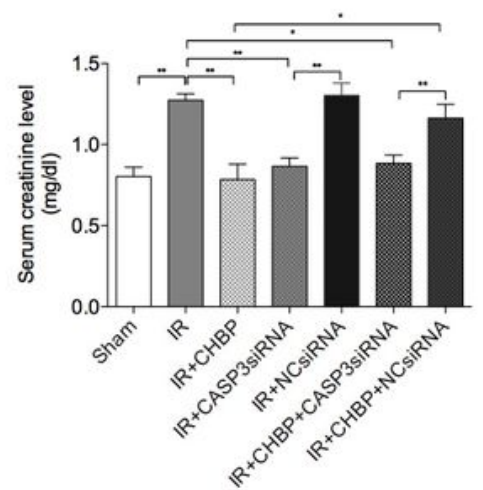

C

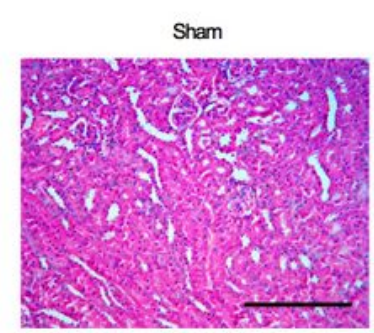

IR

$\mathbb{R}+$ CHBP
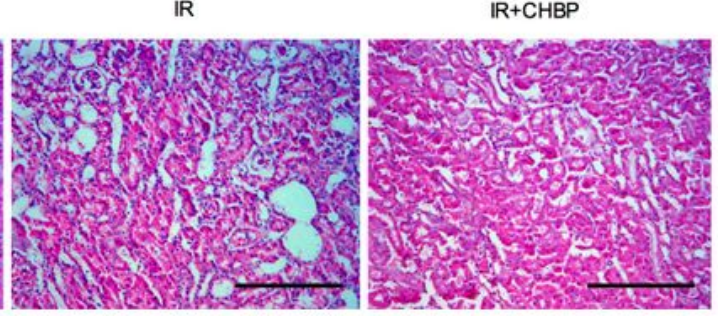

IR+CASP3SiRNA

$\mathbb{R}+\mathrm{NCsiRNA}$

$\mathbb{R}+$ CHBP+CASP3siRNA
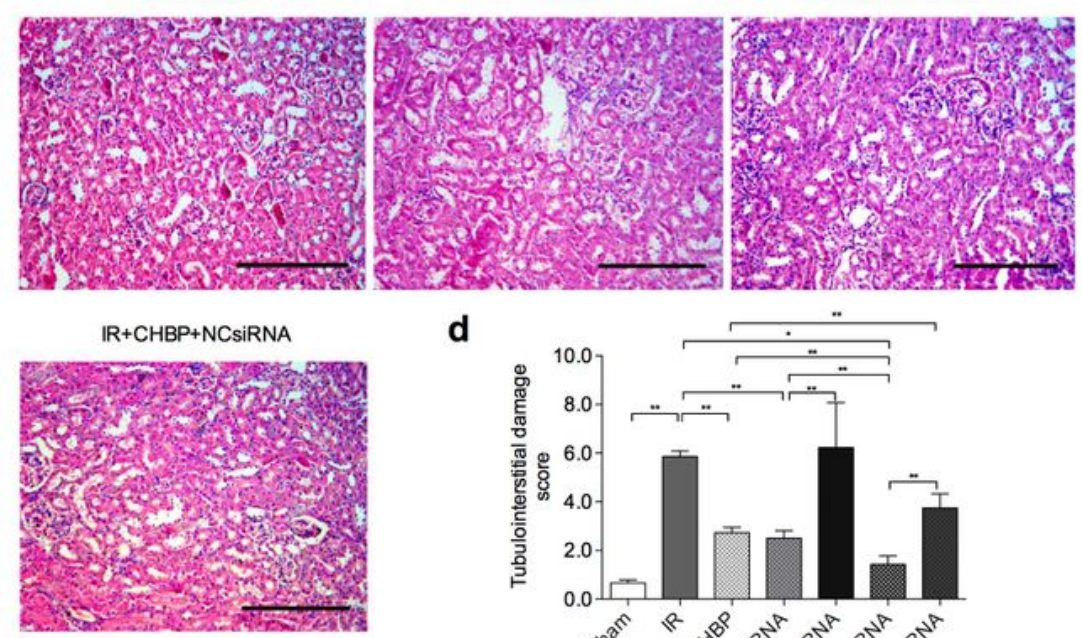

d

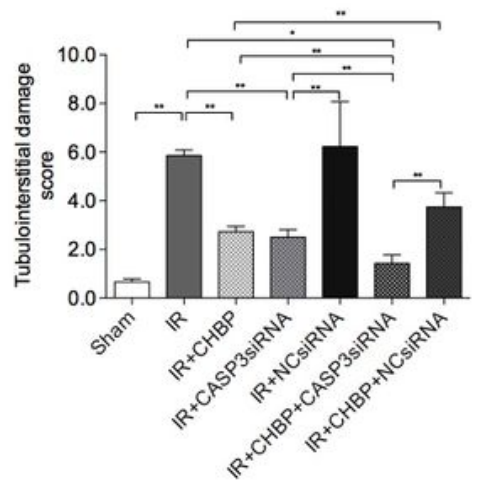

\section{Figure 1}

CHBP and/or CASP3siRNA preserved renal function and structure in IR kidneys. (a) Schematic diagram of mouse renal IR models with the treatment of CHBP and/or CASP3siRNA. Bilateral kidney pedicles were occluded for $30 \mathrm{~min}$, followed by 48 -h reperfusion. CASP3siRNA or NCsiRNA was injected via the tail vein at a dose of $0.03 \mathrm{mg} / \mathrm{kg}$ body weight (BW) $2 \mathrm{~h}$ before surgery. CHBP was given through intraperitoneal cavity at $24 \mathrm{nmol} / \mathrm{kg} \mathrm{BW} 15$ min post reperfusion. (b) The level of serum creatinine (SCr) was 
demonstrated for each group $(n=6)$. (c) Representative photomicrographs of hematoxylin \& eosin $(H \& E)$ staining in renal cortex were shown for each group. Scale bar: $100 \mu \mathrm{m}$. (d) Semi-quantitative analysis of tubulointerstitial damage (TID) score $(n=6)$. * $P<0.05$; ** $P<0.01$.

a

Sham

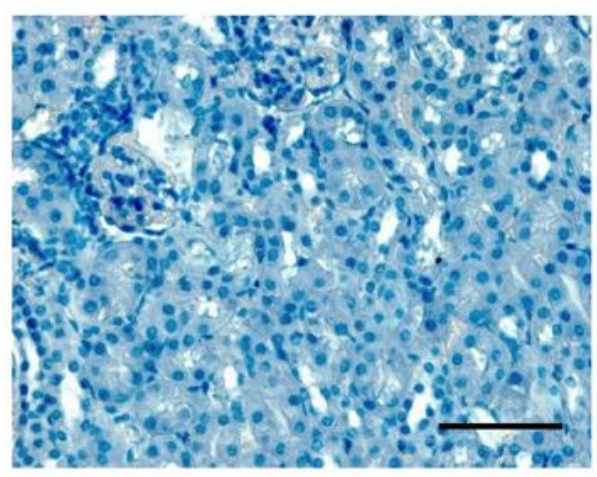

IR+CASP3siRNA

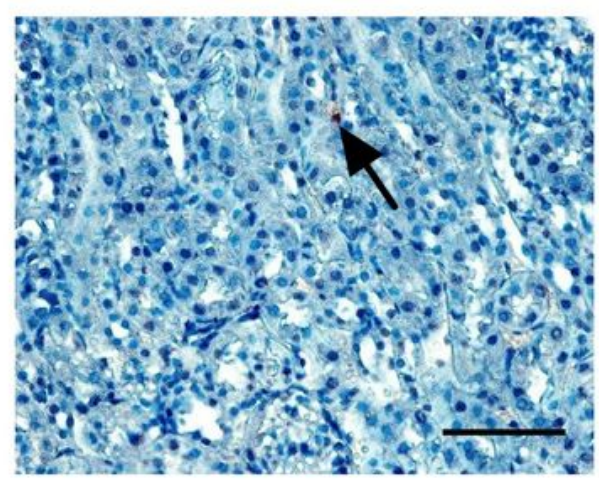

$\mathrm{IR}+\mathrm{CHBP}+\mathrm{NCsiRNA}$

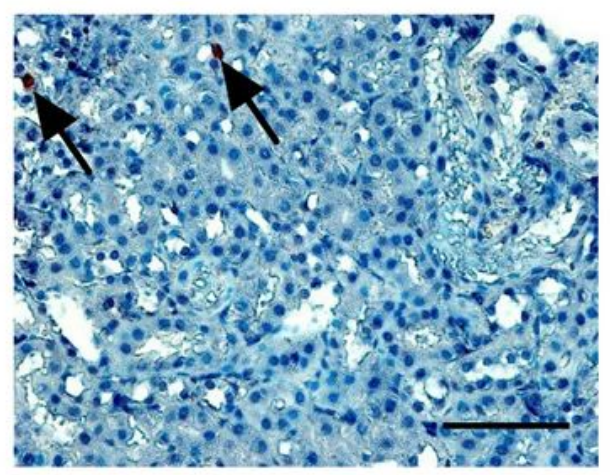

IR

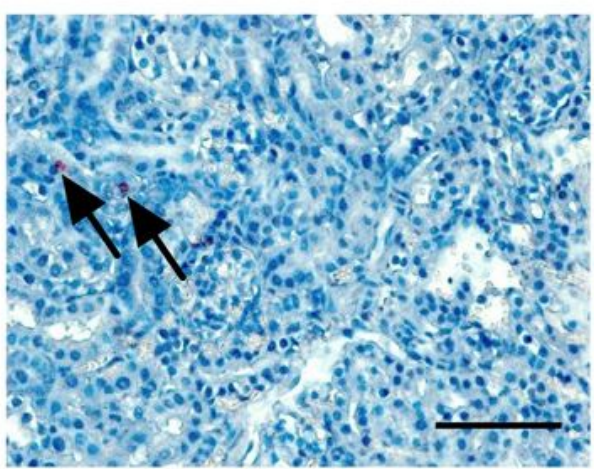

IR+NCsiRNA

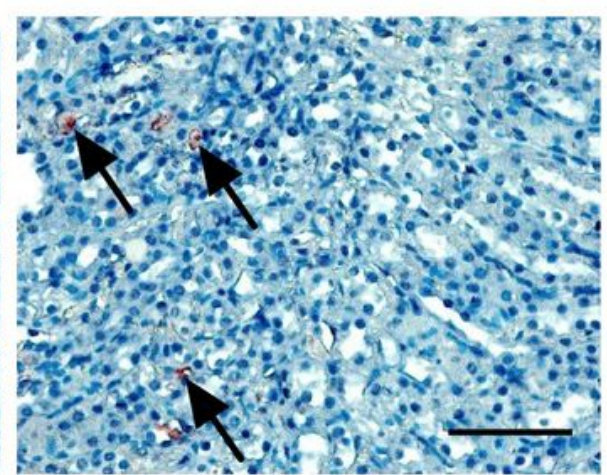

$\mathrm{IR}+\mathrm{CHBP}$

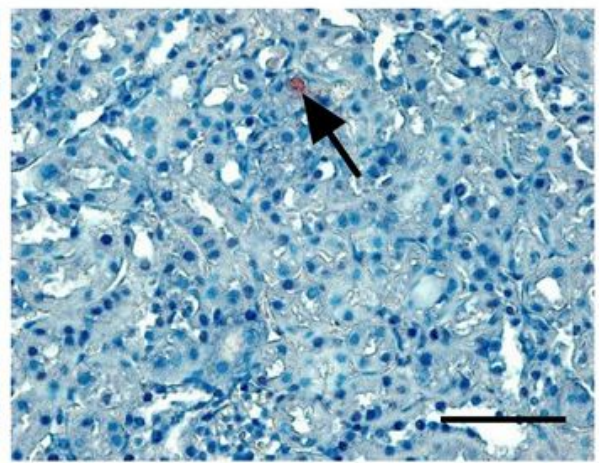

IR+CHBP+CASP3siRNA

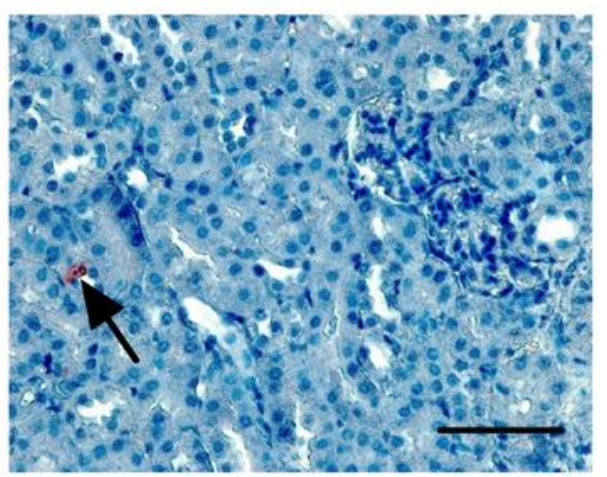

b

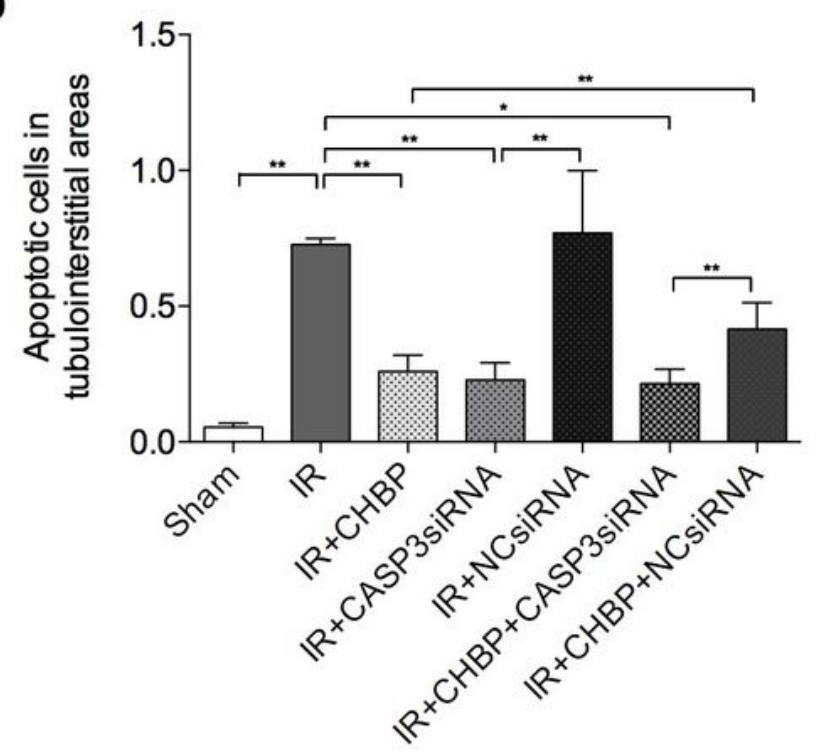

Figure 2

CHBP and/or CASP3siRNA ameliorated apoptosis in tubulointerstitial areas. (a) The method of In situ end labeling (ISEL) fragmented DNAs was used to detect apoptotic cells in kidney tissues. AEC (3-amino- 
9-ethylcarbazole) was used to develop color labelling. Representative photomicrographs of apoptotic cells (indicated by arrows) in cortical areas were shown in each group. Scale bar: $50 \mu \mathrm{m}$. (b) The average number of ISEL+ cells per field are demonstrated for each group $(n=6) .{ }^{*} P<0.05 ; * * P<0.01$.

a

Sham

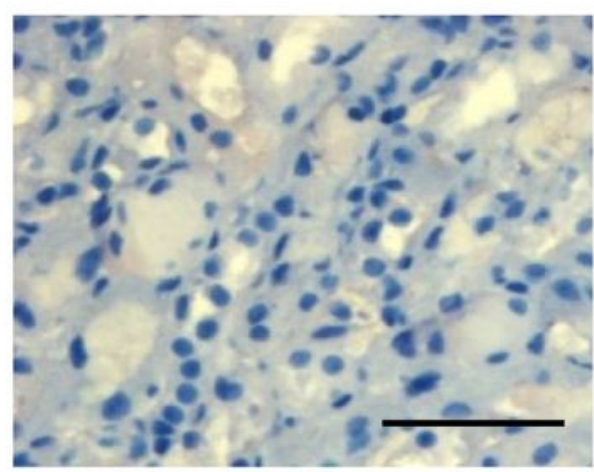

IR+CASP3siRNA

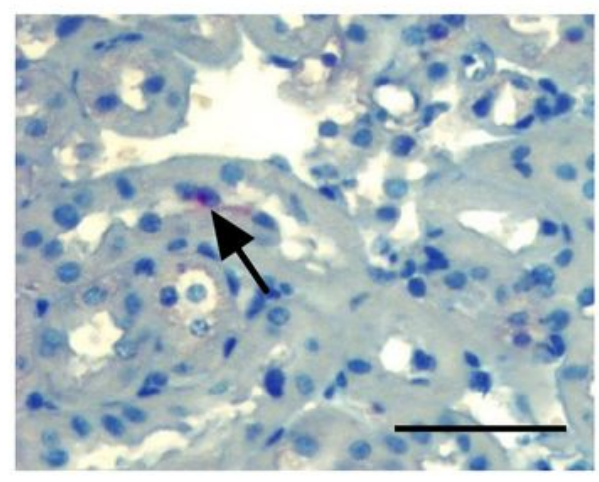

$\mathrm{IR}+\mathrm{CHBP}+\mathrm{NCsiRNA}$

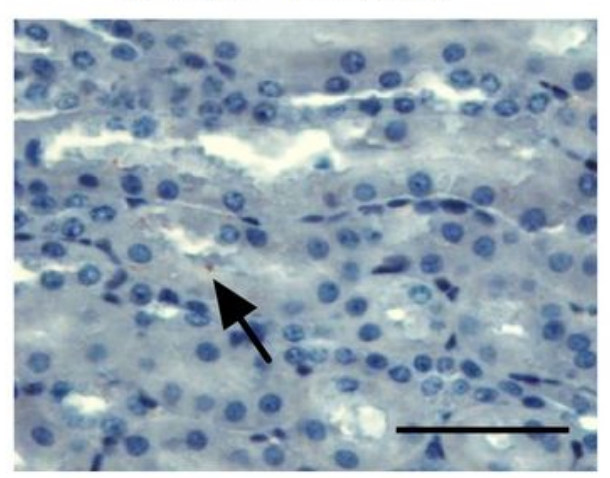

IR

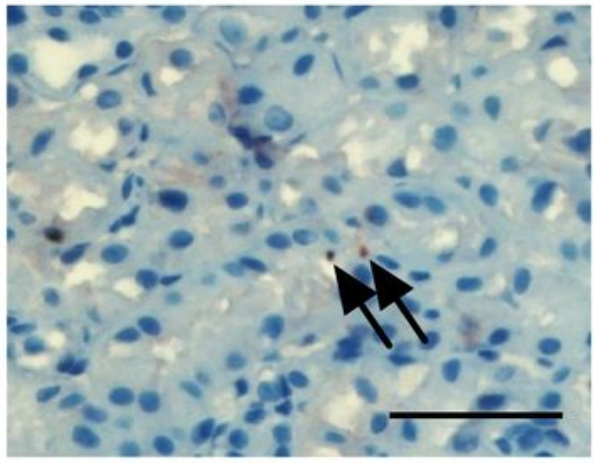

IR+NCsiRNA

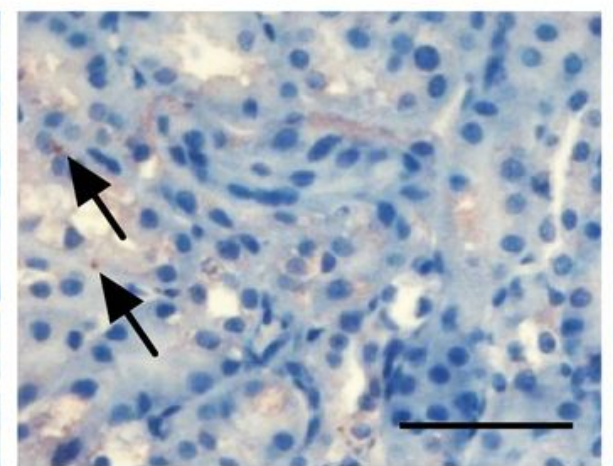

b

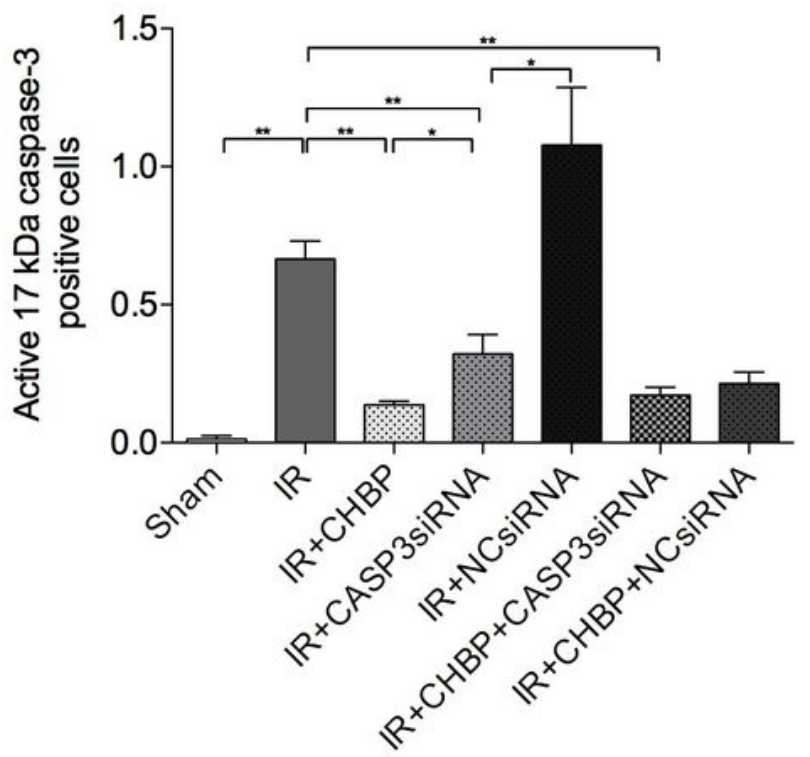

Figure 3

CHBP and/or CASP3siRNA decreased the number of active $17 \mathrm{kDa}$ caspase-3 positive cells. (a) Representative photomicrographs of cells positively stained with $17 \mathrm{kDa}$ caspase-3+ are shown in the 
indicated groups. Detected by immunostaining, $17 \mathrm{kDa}$ caspase- 3 was labeled and revealed by AEC. Scale bar: $50 \mu \mathrm{m}$. (b) The average number of $17 \mathrm{kDa}$ caspase-3+ cells per field are demonstrated in each group $(n=6)$. * $P<0.05 ; * \star P<0.01$.

a
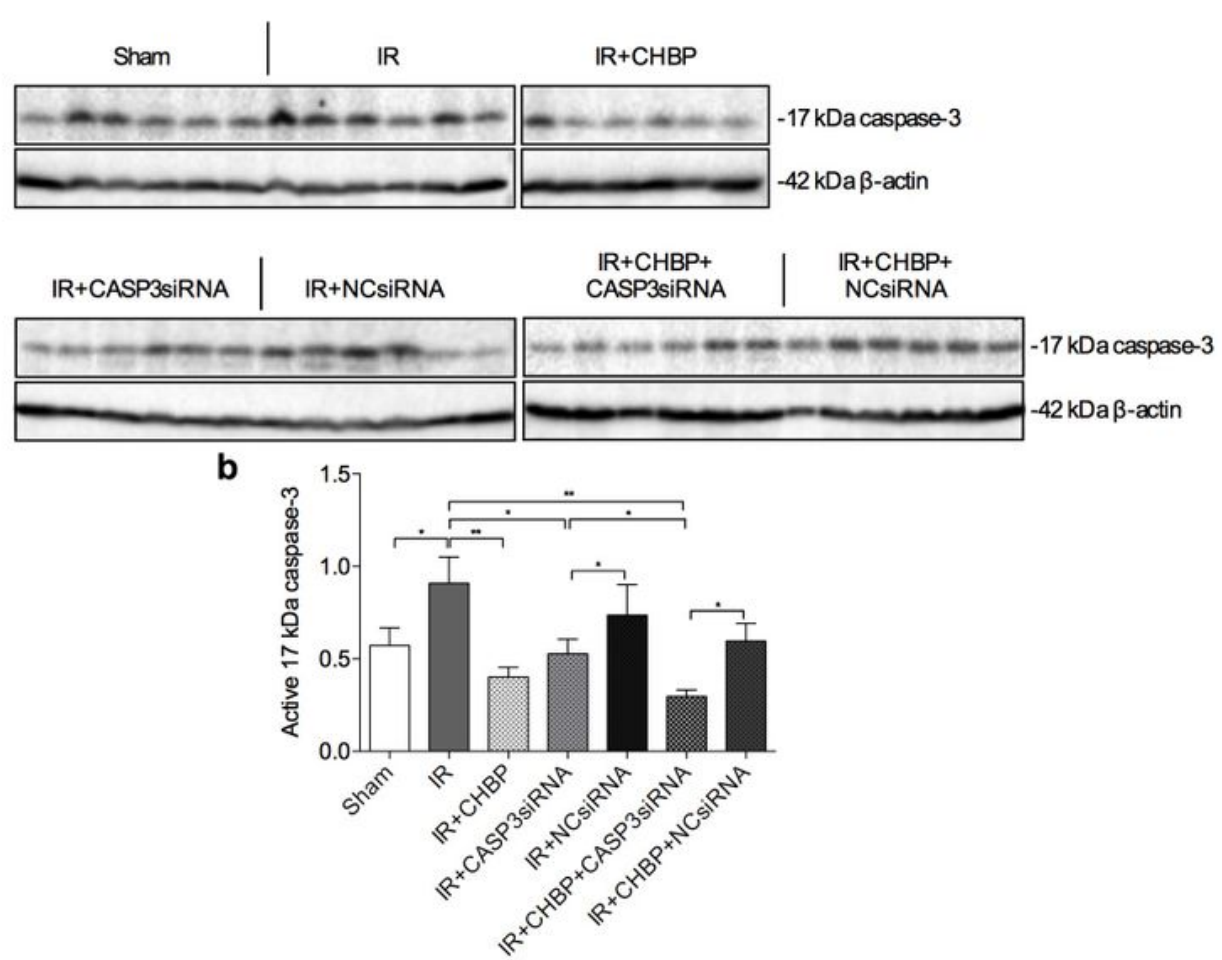

C

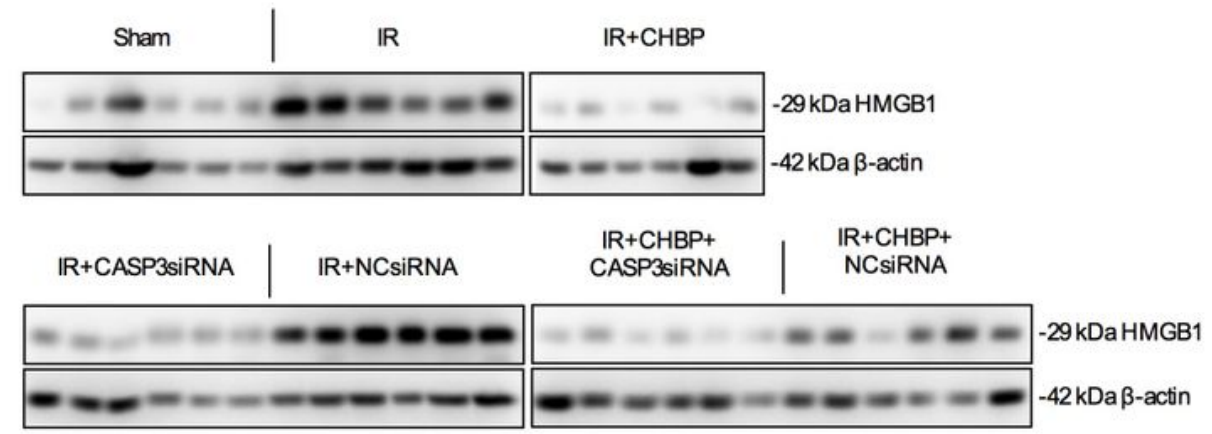

d

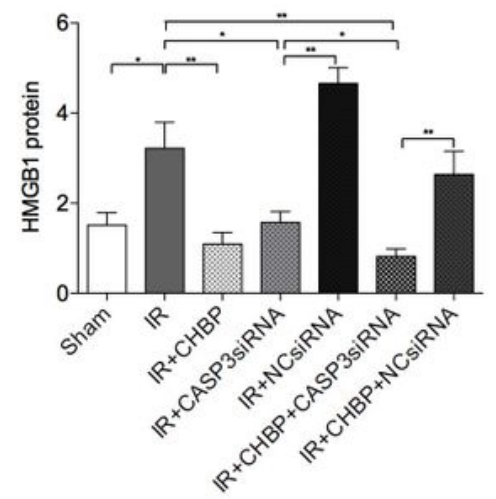

Figure 4

CHBP and/or CASP3siRNA decreased $17 \mathrm{kDa}$ active caspase-3 and HMGB1 expression in IR kidneys. (a) The level of active caspase- 3 was measured by western blotting and typical bands were shown. (b) Semi- 
quantitative analysis showed the expression of $17 \mathrm{kDa}$ caspase-3 corrected by the endogenous control of $\beta$-actin in each group $(n=6)$. (c) Representative bands of HMGB1 were shown. (d) The level of HMGB1 protein corrected by $\beta$-actin was determined in each group $(n=6)$. * $P<0.05$; ** $P<0.01$.

a

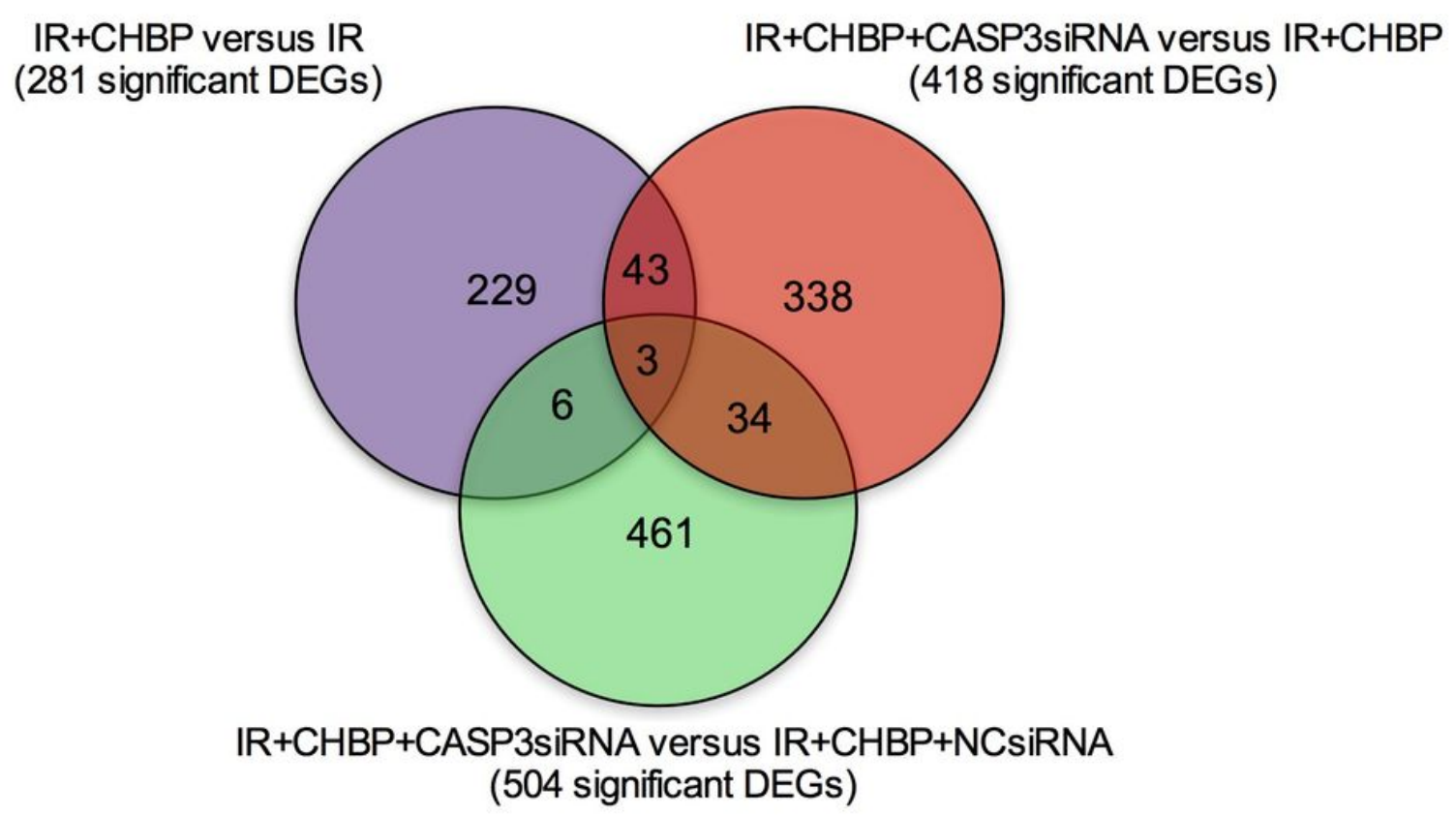

b

C

d

e
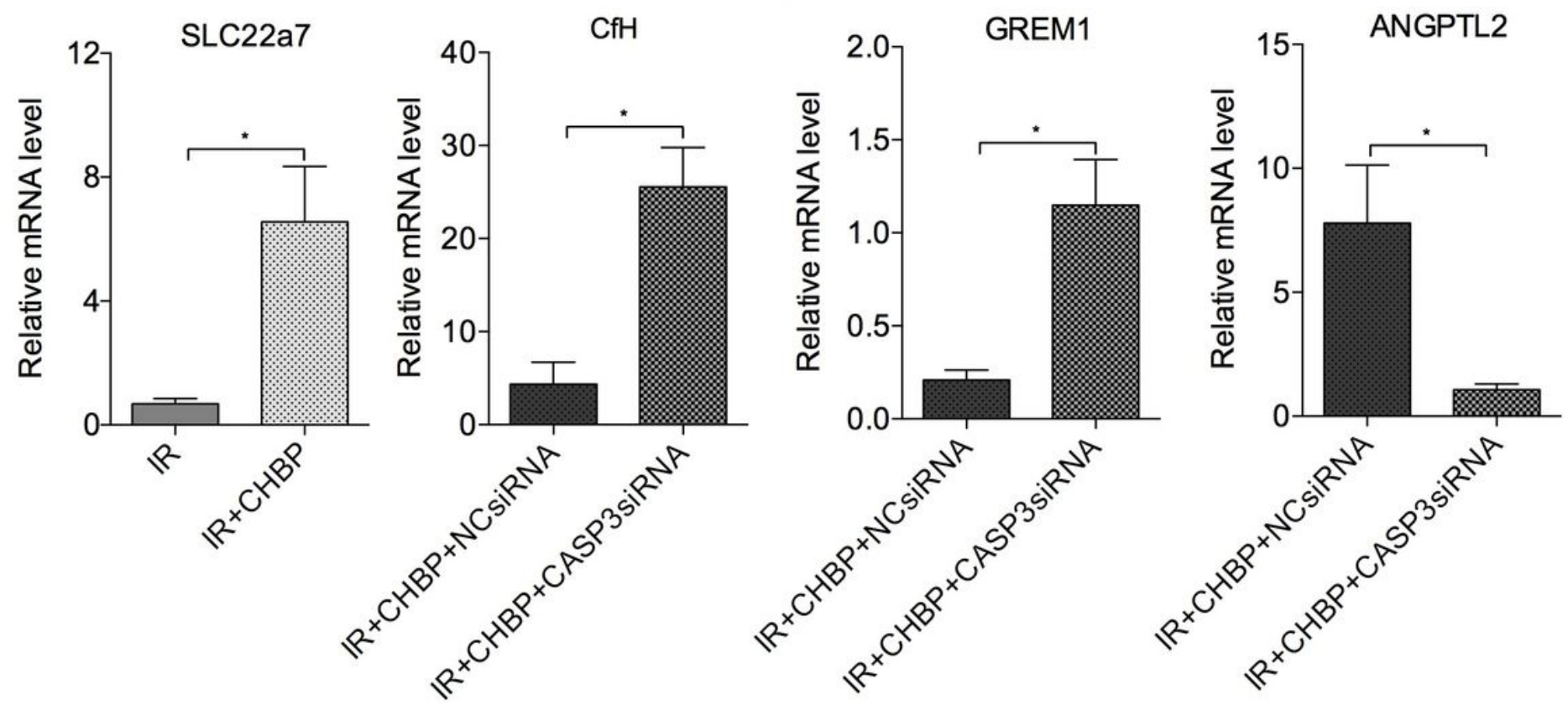

Figure 5

Identified differentially expressed genes (DEGs) and qPCR validation. (a) Venn diagram illustrating the number of significant differentially expressed genes (DEGs) in the three comparisons. The sorting criteria was fold change $(F C)>1.414$ and $P<0.05, n=3$ in each group. (b-e) The expression of SLC22A7, CFH, 
GREM1 and ANGPTL2 mRNA was detected by quantitative polymerase chain reaction (qPCR). SLC22A7: solute carrier family 22 member 7; CFH: complement factor $\mathrm{H}$; GREM1: gremlin 1; ANGPTL2: angiopoietinlike 2. *: $\mathrm{P}<0.05, \mathrm{n}=3$ in each group.

a

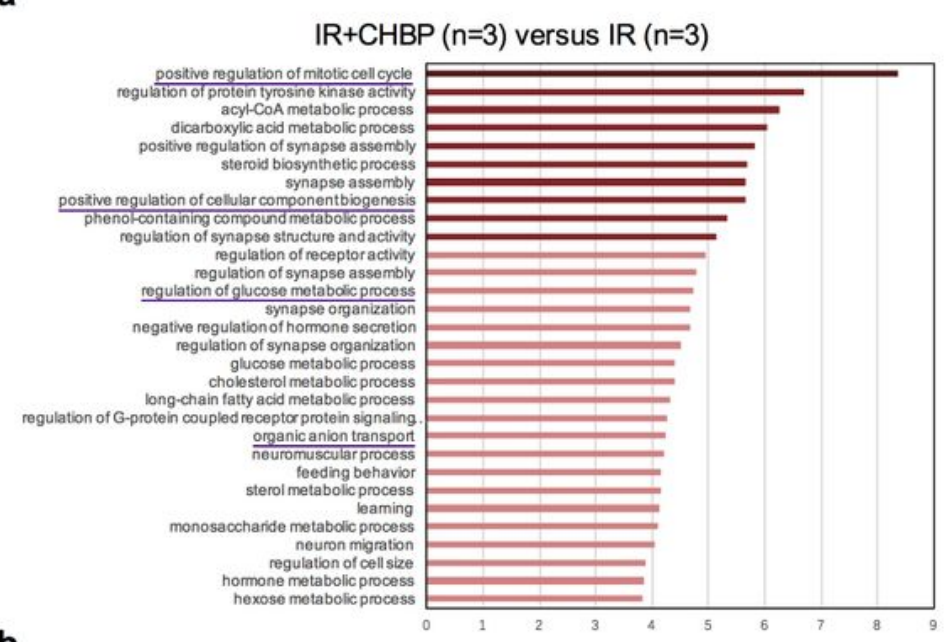

b

$\mathrm{IR}+\mathrm{CHBP}+\mathrm{CASP} 3 \operatorname{siRNA}(\mathrm{n}=3)$ versus $\mathrm{IR}+\mathrm{CHBP}(\mathrm{n}=3)$

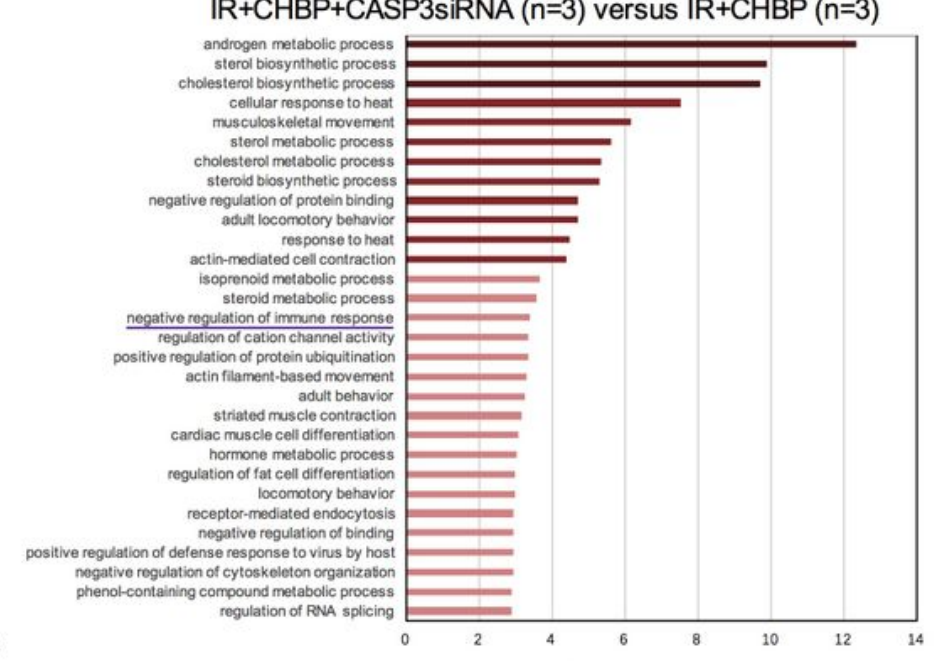

C

$I R+C H B P+C A S P 3 s i R N A(n=3)$ versus IR+CHBP+NCsiRNA $(n=3)$

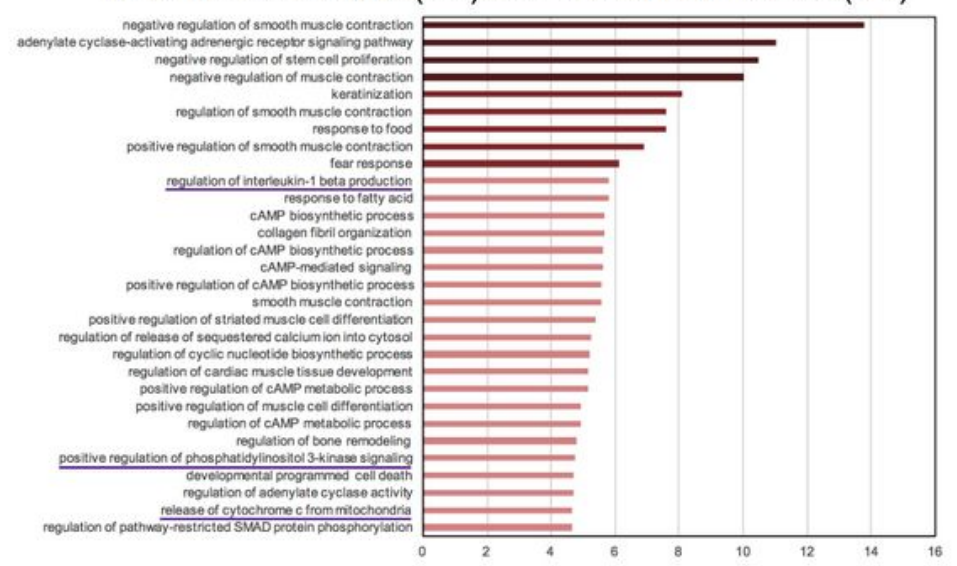

Figure 6

Gene Ontology (GO) analysis of DEGs in IR kidneys. The top 30 significantly enriched GO items of biological processes were shown from 3 comparisons, modified by CHBP (a), and further by CASP3siRNA 
(b) or NCsiRNA (c). The text on the left indicated the category of GO, and the bar chart indicated the enrich factor in each category. Underlined categories were particularly discussed in this study. $n=3$ in each group. 